\title{
A baseline for context-aware system for safety messages dissemination in VANETs
}

Línea de base para sistema sensible al contexto para la diseminación de mensajes de seguridad en VANETs

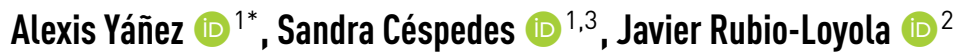 \\ ${ }^{1}$ Departamento de Ingeniería Eléctrica, Universidad de Chile. Av. Tupper 2007. C. P. 8370451 . Santiago, Chile. \\ ${ }^{2}$ Centro de Investigación y de Estudios Avanzados del Instituto Politécnico Nacional (CINVESTAV), Unidad Tamaulipas. Km. 5.5 carretera \\ Ciudad Victoria-Soto La Marina. C. P. 87130. Tamaulipas, México. \\ ${ }^{3}$ Universidad Icesi. Calle 18 \# 122-135 Pance. C. P. 760031. Cali, Colombia.
}

\section{CITE THIS ARTICLE AS:}

A. Yáñez, S. Céspedes and J. Rubio, "A baseline for context-aware system for safety messages dissemination in VANETs" Revista Facultad de Ingeniería Universidad de Antioquia, no. 93, pp. 9-18, Oct-Dec 2019. [Online].

Available:

https://www.doi.org/

10.17533/10.17533/

udea.redin. 20190402

\section{ARTICLE INFO:}

Received: October 26, 2018 Accepted: March 20, 2019 Available online: April 26, 2019

\section{KEYWORDS:}

Vehicular ad-hoc networks, dissemination mechanisms, safety messages, simulation scenario, intelligent transportation systems

Redes vehiculares ad-hoc, mecanismos de diseminación, mensajes de seguridad, escenarios de simulación, sistemas de transporte inteligentes
ABSTRACT: Vehicular ad-hoc networks (VANETs) are mobile networks with highly dynamic contexts of operation. The design and application of context-aware systems could be critical to enhance the performance of protocols deployed in VANETs, which depend on both network and traffic conditions, to detect hostile communication environments, as well as to offer a novel way to make decisions in real-time. In this paper, we present a baseline study for the design of a context-aware system for dissemination of safety messages in VANETs. We model a realistic vehicular intersection and perform extensive simulations to evaluate two well-known dissemination mechanisms, namely the Slotted 1-persistence and the Traffic Adaptive Data Dissemination (TrAD) Protocol, with a set of parameters according to different operation scenarios. We show how the dissemination mechanisms' performance could be improved, or worsened, by choosing a different set of parameter values. By characterizing the operation scenarios, it is possible to adequate the parameters of the dissemination mechanism with the values that work best in a given context, improving in this way the general performance of the dissemination mechanisms under study.

RESUMEN: Los sistemas sensibles al contexto poseen un alto potencial para ser ser utilizados en redes móviles, pues, el contexto en el que operan es altamente dinámico. En particular, las redes vehiculares ad-hoc (VANETs, por su sigla en inglés) se desenvuelven en escenarios donde los sistemas sensibles al contexto podrían ser claves para mejorar el rendimiento de los protocolos que dependen de las condiciones de red y tráfico, para detectar entornos de comunicación hostiles, así como también para ofrecer una forma novedosa de tomar decisiones en tiempo real. En este artículo presentamos el estudio de una línea de base para el diseño de un sistema sensible al contexto para la diseminación de mensajes de seguridad en VANETs. Se modela de manera realista una intersección vehicular y se simulan extensivamente dos mecanismos de diseminación bien conocidos; el Slotted 1-persistence y el Traffic Adaptive Data Disemination (TrAD) Protocol, con conjuntos de parámetros según diferentes escenarios de operación. Los resultados demuestran cómo el rendimiento de los mecanismos de diseminación podría mejorar o empeorar al elegir un conjunto diferente de valores de parámetros. Al caracterizar los escenarios de operación acertadamente, es posible adecuar los parámetros de un mecanismo de diseminación con los valores que funcionan mejor en un contexto dado, mejorando de esta manera el desempeño general de los mecanismos estudiados.

\section{Introduction}

The Vehicular Ad-hoc Networks (VANETs) have emerged

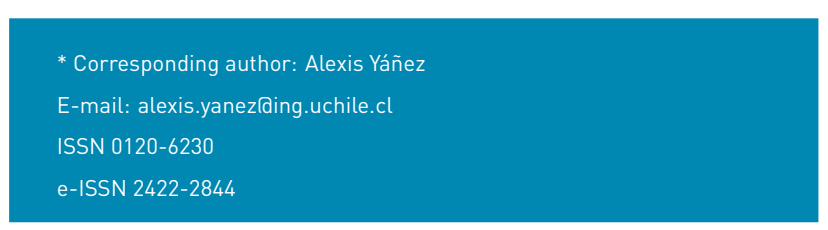

as one of the most promising solutions to reduce traffic accidents and improve the efficiency of the Intelligent Transportation Systems ITS. However, thanks to the development of technologies that allow connectivity in vehicular environments, a variety of applications have been proposed that can use this type of network with different objectives, for example; sending alerts to people about real-time road hazards to avoid the most common 
traffic accidents [1, 2], allowing the transfer of large quantities of data for entertainment applications such as music, video, and Internet access, among others [3].

Regarding safety applications, these can significantly reduce the number of accidents on the route. For example, it is possible to reduce the number of vehicular collisions by a $60 \%$ thanks to the Forward Collision Warning (FCW) application [4]. Safety applications are based on dissemination mechanisms that allow delivering one or more alert messages to vehicles that are within the area of influence of an event. In ad-hoc mobile networks, the dissemination mechanisms generally do not use acknowledgment messages $(A C K)$, so it is usually not possible to detect packet losses when there are poor conditions in the communications network [5]. In VANETs, it is necessary not only to deliver emergency messages with the least possible delay, but also to guarantee the reliability of the delivery, so the design of dissemination protocols constitutes a challenging problem for the successful deployment of safety applications [6].

One of the key challenges when designing dissemination protocols is the fact that vehicular environments should be characterized as highly dynamic contexts, with conditions that vary depending on the type of route, time of day, location, and traffic density, among other parameters.

Depending on the application's operation, the dissemination is performed only to neighboring vehicles (i.e., single-hop) or to all vehicles that are in the area of interest via multi-hop dissemination [7]. A very common problem taking place during dissemination is the so-called broadcast storm problem [8], which occurs when many vehicles send information at the same time.

In networks with a high vehicular density, i.e., with a high number of nodes in the VANET, a large number of packet collisions occur, especially when a broadcast storm is in place, causing a decrease in the quality of communications and the impossibility of delivering the emergency messages of the dissemination protocol. By knowing the characteristics of the operation scenario, the dissemination mechanisms may take advantage of the knowledge obtained from the environment to set the appropriate parameter values, and in turn, this may improve the performance metrics of the protocol. However, in ad-hoc networks, it is assumed there is no centralized database where to store reports about the state of the communications network, nor the state of the vehicular traffic. Given a particular VANET node, it is not trivial to determine the general context in which the running protocols and applications operate.

In this paper, we extend our preliminary results presented in [9] and provide a baseline study for the design of a context-aware system for dissemination of safety messages in VANETs.

This study consists in evaluating the dissemination mechanisms using metrics that allow us to measure their performance as well as to compare the adjustment of their parameters (or among the mechanisms) under different joint conditions of both, the communications network and the road traffic. With this information, it will be possible to define the set of parameters that work best for a given scenario. Although most of the dissemination mechanisms are parameterizable, the lack of knowledge of the environment in which they operate makes it difficult for the protocols to adapt to the actual conditions of the environment. This work serves as an input for a future system that will classify, in a decentralized manner, the type of scenario in which the dissemination protocol must operate, so that its parameters can be adjusted to the operating environment.

The remainder of the paper is organized as follows: Section 2 describes previous works related to data dissemination mechanisms in VANETs and works that propose the use of context-aware systems in vehicular environments. Section 3 discusses the results of our baseline study with the Slotted 1-persistence dissemination mechanism and the Traffic Adaptive Data Dissemination (TrAD) Protocol; it includes the methodology for characterizing the scenarios of simulation and the discussion of the results. The concluding remarks and future work are presented in Section 4.

\section{Related work}

\subsection{Data Dissemination Mechanisms}

The exchange of data between vehicles is an important part of applications deployed in VANETs, and its management has a real impact on their performance and functionality. When deployed on mobile and heterogeneous environments, in circumstances of high or low intensity of network traffic, the way in which data is disseminated faces different challenges. Here we present a body of research work that has been devoted to solve the problem of the exchange and propagation of relevant safety information in a vehicular network.

The concept of data dissemination often refers to data-casting, which can be seen as a limited and controlled way in which data flows through the network [10]. Unlike routing algorithms, which seek to discover a route to deliver information between two nodes, data dissemination strategies are employed to deliver a message to all nodes or a particular group, located in a specific geographical area. Therefore, data dissemination takes into account 
the network load and at the same time it must ensure to deliver data to nodes that need it, with reduced latency, to facilitate the decision-making process in a timely manner.

In the past, there have been schemes and protocols for dissemination that rely on the infrastructure or on an ad-hoc network [11, 12]. The review in this section focuses only on works that fall on the ad-hoc category for dissemination, which resembles a VANET. A widely used mechanism for dissemination is the data delivery over multi-hop communications, where data is disseminated using intermediate nodes as relays [7]. However, a prevalent problem in the ad hoc networks is the so-called "broadcast storm" [8], which occurs when many vehicles send packets at the same time. In the presence of a large number of sources of traffic, packet collisions occur, decreasing quality in communication and generating data losses. In a VANET, such a situation causes that critical events, like near car accidents, are not disseminated on time. Therefore, the poor performance of the communications network represents a severe problem for the safety of users in the vehicular network, especially if we consider that a prompt reaction to risky events depends on the timely delivery of safety information. The problem of a congested communications network is usually related to a highly dense vehicular network traffic, which is a recurrent scenario in urban environments. The work in [13] is designed to avoid "broadcast storms" in vehicular scenarios.

In multi-hop dissemination, there are several proposals, some aimed at making the forwarding opportunistically [14]; other approaches employ genetic algorithms to choose the best position to perform multi-hop transmission, looking for the optimum configuration parameters with other methodologies such as fuzzy sets based on rules [15]. There are also protocols that consider scenarios of distant vehicles where the stability of network connectivity is very low. This area of study is known as Delay Tolerant Networks (DTN), which exploit opportunities for encounters in partitioned networks [16, 17]. In addition, the frequent partitions in VANETs are not only because nodes may be far away, but also due to obstacles that impede line of sight and produce adverse effects such as shadowing [18, 19], generating temporary disconnections among nodes.

Furthermore, some protocols establish a scheme to deal with scenarios with different vehicular traffic conditions [20, 21], as well as schemes dedicated to events of interest in urban environments [22]. Other works focus on providing reliable accident warnings on the route [23] or they are designed for specific roads, such as intersections [5]. An example of reliable dissemination designed only to work on the first hop is described in [24].
To address the problem of having different scenarios in the topology of VANETs, various forms of one-hop dissemination have been proposed. Such proposals for "adaptive beaconing" $[25,26]$ use the operating conditions to adapt the beacon rate, transmission power, and the transmission channel. An innovative proposal focused on adapting the same parameters using the variation of acceleration to decide how the "beaconing" mechanism is executed is described in reference [27]. According to the parameters that the adaptive protocols modify, these can be classified into the following general categories [28]:

\section{Message frequency control (MFC)}

\section{Transmit power control (TPC)}

\section{Miscellaneous (Multichannel Switch)}

\section{Hybrid. (Combination of the above)}

Regarding the evaluation of the dissemination mechanisms, one can take into account different parameters to compare the effectiveness/efficiency among the protocols. Some of the most popular metrics used are the normalized routing load, packet losses, transmission rate, and latency. In [29], a comparative study of dissemination protocols using "broadcasting" is presented. Although not very relevant for VANET scenarios, the energy efficiency has also been studied for some dissemination mechanisms to be able to implement them on intelligent transport systems that integrate other types of energy-efficient vehicles [30].

\subsection{Context-aware Systems}

Context-aware systems are characterized by being aware or being sensitive of the situation (or context) in which they operate, either in physical, virtual or user's environment [31].

The context is understood as "any information that can be used to characterize the situation of an entity. An entity is a person, place or object that is considered relevant to the interaction between a user and an application, including the user and the applications themselves" [32]. These systems are able to adapt, exploiting the knowledge acquired through sensors, other nodes or through the infrastructure available on the route.

One of the first works related to context-aware systems was presented in [33]. In this article, the authors developed an active tag location system, which based on infrared technology can determine the current location of a user, this was used to forward telephone calls to a telephone near the user. 
In vehicular environments, context-aware systems focus mainly on the area of service recognition and location on the route $[34,35]$. In [32], the authors describe various approaches that have used context-aware systems for the detection of specific events, for example in security, entertainment, and traffic management applications. To the best of our knowledge, there is only one work that has addressed the problem of disseminating information of accidents based on warning messages considering the information of the vehicular context [36]; however, the scheme does not consider the traffic of the communications network in the context definition.

Context-aware routing in VANETs is another area of research related to our work. In [37], the authors present an approach to provide Internet access to support interactive and entertainment applications in VANETs. In [38], the authors employ the source and destination location of nodes as the context information; then, they elaborate a routing mechanism that seeks to reduce packet losses due to saturation in the cache of each vehicle while avoiding network overload. In [39], the authors define the vehicular context based on parameters like speed of each node and inter-vehicular distance. This context information is evaluated and taken into account while selecting the appropriate transmission speed considering the conditions of the link layer. The approach produces important VANET performance enhancements in terms of data transmission rates between vehicles.

In [40], the authors present a classification of different broadcasting mechanisms and their effect in the performance of different VANET applications. The main contribution consists in the differentiation of broadcasting schemes based on client applications. Authors in [41] describe a context-aware system that identifies potential accidents and pro-actively enables safety mechanisms before the accident occurs. The information acquired from the sensors in the vehicles (i.e., data prior to the accident) is analyzed to predict a certain collision and its severity; in this way, the system launches a timely warning. In [42], the authors propose a context-aware system to improve the way in which information is acquired to avoid redundancy in the information collected by a group of nodes, whereas the authors in [43] propose a MAC layer context-aware protocol based on Bayesian networks with the aim at enhancing the performance of the VANET in highly congested scenarios. The approach presented in [43] produces a substantial improvement compared to the CSMA/CA mechanism adopted by the IEEE 802.11-OCB standard, whose performance is seriously affected in highly congested environments.

\section{Message dissemination in VANETs: a baseline}

In this section, we provide a baseline study aiming at demonstrating the effectiveness of adjusting the parameters' values of a dissemination mechanism according to the context where the VANET is operating. The dissemination mechanisms under study are evaluated according to the following performance metrics [21]:

- Packet Delivery Ratio $(P D R)$ : represents the average number of messages received by vehicles within an area of interest. PDR is expressed as a percentage of the total number of messages sent by the source node. This metric is also known as dissemination coverage.

- Delay: is the average of the time it takes since the source node sends a packet until it is received by each vehicle in the area of interest.

- Dissemination speed: represents the speed with which the message is disseminated to vehicles in the area of interest. To obtain this metric, the source node sends the message only once and then the coverage increment must be calculated as a function of time. If the coverage increases significantly in a small period, it means that the dissemination protocol has a good performance with respect to this metric.

\subsection{Dissemination mechanisms chosen for the evaluation}

\section{- Slotted 1-persistence [13]}

Is a dissemination mechanism that gives the priority of re-transmission to the farthest node of the sending node. For that, the protocol uses a waiting time before re-transmitting, which varies according to the distance between nodes. When a node receives a packet, the protocol verifies the packet ID; if the packet is received for the first time and if the node has not received any duplicates before, the protocol assigns a time slot. Then, the node performs a re-broadcast with a probability of 1 within the time slot $T_{S_{i j}}$ assigned. Otherwise, the packet is discarded. The time slot can be obtained as indicated by Equation (1):

$$
T_{S_{i j}}=S_{i j} \times \tau,
$$

where $\tau$ is the estimated delay of a hop, which refers to the sum of the propagation delay plus the delay of accessing the shared medium. $S_{i j}$ is the assigned slot number and is expressed in Equation (2) as follows:

$$
S_{i j}=N_{S}\left(1-\left[\frac{\min \left(D_{i j}, R\right)}{R}\right]\right),
$$


where $D_{i j}$ is the relative distance between the nodes $i$ and $j, R$ is the transmission range, and $N_{S}$ is the default slot number. $N_{S}$ must be chosen as a function of the traffic density. The authors in [27] suggest a value of $N_{S}=5$ in rush-hours or under traffic congestion scenarios. The value $N_{S}=3$ is recommended for other cases. This protocol has been chosen for the study because it solves the problem of broadcast storms and because it needs only the information about the position of the sending and receiving nodes to operate. It is also one of the most widespread used protocols in the literature for its simplicity and because it was one of the first protocols proposed for dissemination.

\section{- The Traffic Adaptive data Dissemination (TrAD) protocol [21]}

This protocol employs adaptive beaconing, so that, based on the scenario in which mobiles nodes operate (urban or highway), the mechanism chooses between two ways of broadcasting that intend to improve the performance of the network. In addition, this mechanism assigns certain roles to the vehicles, depending on their position with respect to a place of interest, specifically when using store-carry-and-forward.

To avoid broadcast storms, TraD assigns different delays to re-transmissions of WAVE short messages (WSM) in the following way [21]: first, the source node establishes a ranking based on the utility function described with Equation (3) expressed as follows:

$$
U_{T X}=\omega_{\mathrm{CBR}}\left(\frac{N+D}{2}\right) \text {, }
$$

which involves the distance between sender and receiver $D$, the number of neighbors $N$ of a given node, and the channel busy ratio $C B R . \quad D$ is calculated with Equation (4) expressed as follows:

$$
D=\min \left(\frac{\text { Dist }_{\text {sn }}}{\text { max.RadioRange }}, 1\right)
$$

where ist $_{s n}$ is the distance between the sender and its neighbor and max.RadioRange is the maximum communication range over the wireless link.

$N$ is calculated with Equation (5) expressed as follows:

$$
N=\min \left(\frac{\# N \text { eighbor }}{\max . \# N \text { eighbor }}, 1\right) \text {, }
$$

where \#Neighbor is the number of neighbors in the vicinity and $\max$.\#Neighbor is a pre-set maximum number of neighbors.

The $\omega_{\mathrm{CBR}}$ is defined as a piece-wise linear function in terms of the CBR. It is defined in Equation 6 as follows:

$$
\omega_{\mathrm{CBR}}= \begin{cases}1 & 0<\mathrm{CBR}<0.6 \\ 1-\mathrm{CBR} & 0.6 \leq \mathrm{CBR}<0.8 \\ 0.001 & 0.8 \leq \mathrm{CBR}<1\end{cases}
$$

The sending node collects the aforementioned information via beacons, i.e., each vehicle appends it own information before sending a beacon message. With this, a sender node sends the ranking to its neighbors. Then, upon reception of a safety message, the receiving node checks the ranking and assigns the delay for re-transmission Delay $X$. According to [21], Delay $y_{T X}$ is calculated in Equation (7) as:

$$
\operatorname{Delay}_{T X}=R \cdot t i
$$

where $R$ corresponds to the position in the generated ranking. This protocol is chosen for the study since it takes into account the performance of the communications network as well as the state of the vehicular traffic.

\subsection{Methodology and Characterization of scenarios}

To obtain the baseline results, we employed the intersection illustrated in Figure 1, which is deployed on an intersection of two avenues with high vehicular traffic in the city of Santiago, Chile. The intersection has 3 and 4 lanes per road. The map is set in the SUMO simulator and we introduced vehicles according to 2 different densities: low $15.6[\mathrm{veh} / \mathrm{km} / \mathrm{ln}]$ and high $73.4[\mathrm{veh} / \mathrm{km} / \mathrm{ln}]$. The densities seek to replicate representative values within fuzzy sets reported in [44], where the authors classify different vehicular traffic density degrees, according to real measurements carried out in [45]. With the objective to observe the performance at critical levels, we chose the extreme densities (low and high), which are consistent with the density values employed for the protocols under evaluation in their original proposal [13].

The scenario is evaluated with different configurations of the following network parameters:

- Beaconing frequency varying between $2 \mathrm{~Hz}$ and 10 $\mathrm{Hz}$. These messages carry mandatory information for the operation of safety applications, such as position, velocity, and acceleration of the vehicle. 


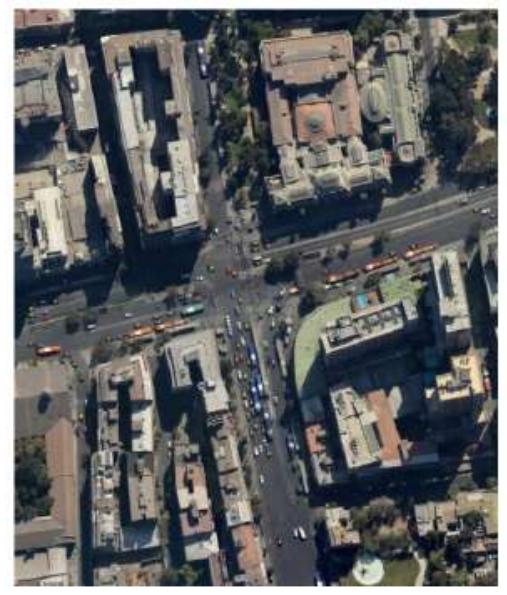

(a) Satellite image

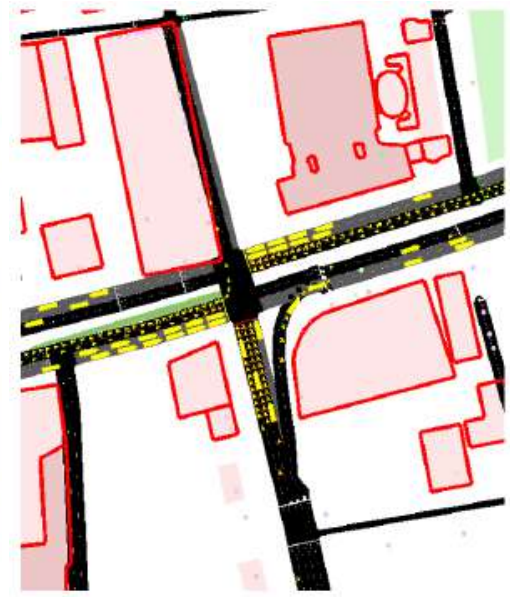

(b) Sumo Scenario

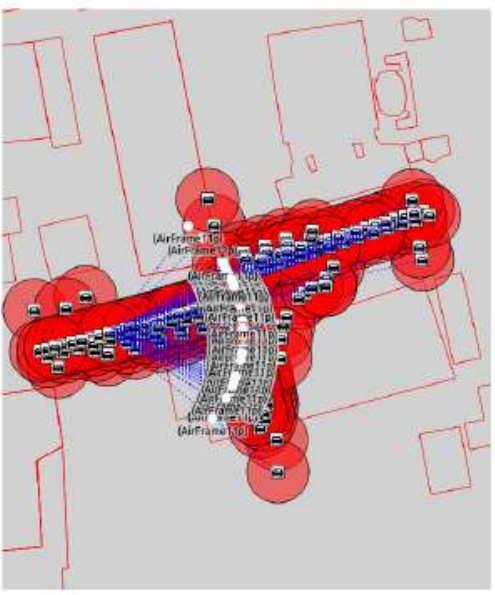

(c) OmNet++ Scenario

Figure 1 Views of scenario

- Wave Service Advertisement messages (WSA) with frequency of $1 \mathrm{~Hz}$. These messages are included to simulate background traffic on the network corresponding to various on-route services (i.e., traffic from other safety applications). They are additional load to the beaconing that operates permanently.

Table 1 Network loads definition

\begin{tabular}{ccc}
\hline $\begin{array}{c}\text { Network } \\
\text { load }\end{array}$ & $\begin{array}{c}\text { Beaconing } \\
\text { frequency }\end{array}$ & WSA \\
\hline 1 & $2[\mathrm{~Hz}]$ & no \\
2 & $10[\mathrm{~Hz}]$ & no \\
3 & $10[\mathrm{~Hz}]$ & yes \\
\hline
\end{tabular}

The labeling of each scenario according to the existent load in the communications network is listed in Table 1. The loads were chosen according to the common frequency values employed in active road safety and cooperative navigation applications reported in [46]. To understand the response of the mechanisms under study when there is an increase in network traffic load, an additional load coming from the WSA messages is added to the channel. Such a load is only included for the $10 \mathrm{~Hz}$ configuration to recreate extreme conditions of congestion over the channel.

During the simulation, a notification message for dissemination is generated only once by the source vehicle, that is, only one packet of type Wave Short Message is generated to observe its behavior at the first instant of dissemination. The packet is disseminated at time instant $t=5 \mathrm{~s}$ when an accident over the intersection triggers the safety notification message. The message is forwarded by the neighboring nodes across the entire region of interest (ROI). Because of this, when the PDR is calculated, this percentage is also a representation of the number of nodes in the ROI that received the message successfully.

We executed 20 runs for each configuration scenario, for a total of 320 simulation runs. Table 2 shows the details of the parameters used in the bidirectional simulator VEINS [47] employed for this evaluation.

\subsection{Discussion of results}

To begin our discussion, we examine the Slotted 1-persistence's performance; it should be noted that to the best of our knowledge, the effects of other values of $N_{s}$ (see Equation 2) have not been evaluated in the literature for particularly dense scenarios. The values $N_{s}=3$ and $N_{s}=5$ are the ones suggested by the authors of this mechanism for low- and high-density scenarios, respectively [13]. In this section, we present an evaluation of the protocol's performance considering two additional values of $N_{s}$; in low density scenarios we employ $N_{s}=2$ and $N_{s}=3$; in high density scenarios we employ $N_{s}=5$ and $N_{s}=7$.

In the case of a low density, we can observe in the results in Figure 2 the different behaviors of the protocol for different network loads. In particular, Figures $2 \mathrm{a}$ and $2 \mathrm{~b}$ show that when the network load is high (i.e., $10 \mathrm{~Hz}$ of beaconing frequency and other WSA background messages) the PDR is reduced by almost a $40 \%$ and the end-to-end delay increases by almost a $200 \%$. Although it seems the dissemination speed, shown in Figure 2c, increases in the same scenario, it has to be analyzed together with the PDR, i.e., most likely there is a high dissemination speed but for a reduced number of successfully delivered 


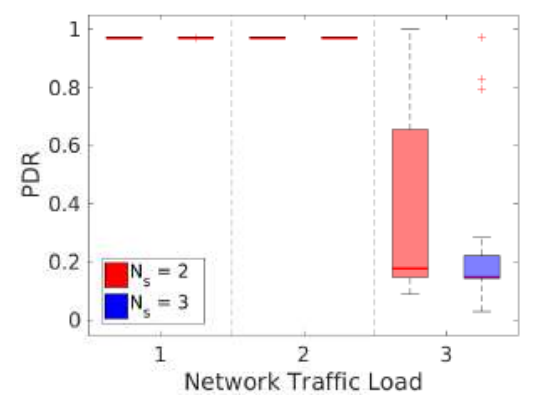

(a) Packet delivery ratio

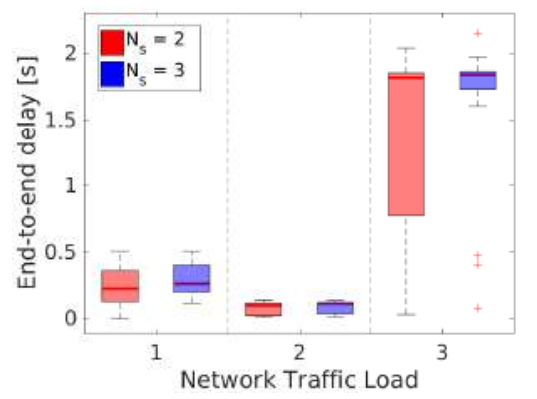

(b) End to end delay

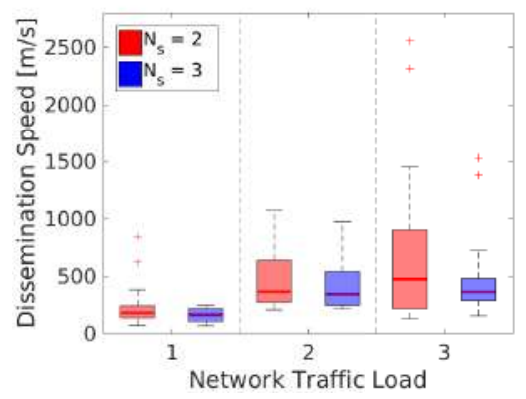

(c) Dissemination speed

Figure 2 Slotted 1-persistence evaluation: different network loads and low vehicular density $\lambda=15.6[\mathrm{veh} / \mathrm{km} / \mathrm{ln}]$

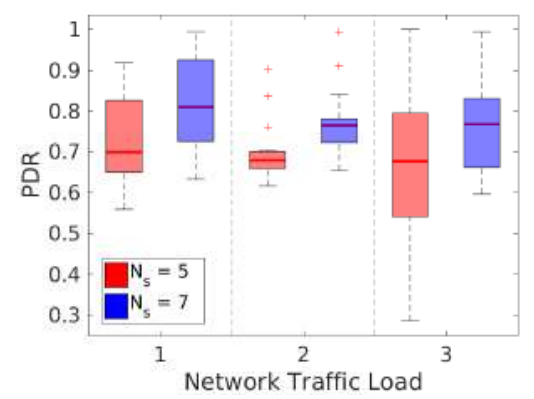

(a) Packet delivery ratio

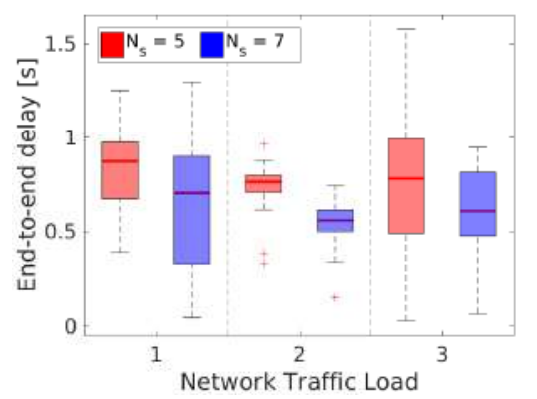

(b) End to end delay

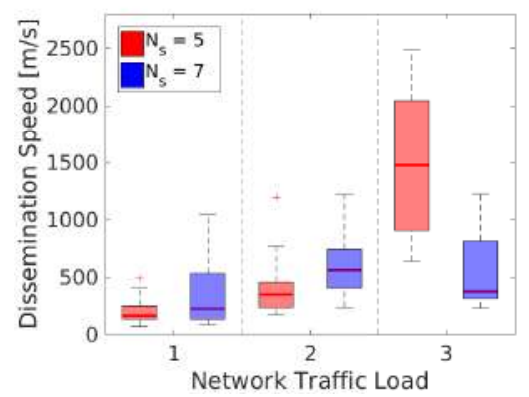

(c) Dissemination speed

Figure 3 Slotted 1-persistence evaluation: different network loads and high vehicular density $\lambda=73.4[\mathrm{veh} / \mathrm{km} / \mathrm{ln}]$

packets. Also, one could suggest that using $N_{s}=2$ is better for any given context with a low vehicular density, independent of the network load. Although the average for PDR, end to end delay, and dissemination speed are very similar in both $N_{s}=2$ and $N_{s}=3$, it is shown that data dispersion is better in $N_{s}=2$.

In the case of high density, one can observe in Figure 3 that when employing $N_{s}=7$ instead of $N_{s}=5$ it may result in a better protocol's performance when the dense network is combined with network load configurations 1 and 2 . In such scenarios, for $N_{s}=7$, the PDR shown in Figure $3 \mathrm{a}$ increases around a $10 \%$ in average, with a reduced end to end delay and an higher dissemination speed (see Figures $3 b$ and $3 c$ ). However, in the case of network load configuration 3 , the dissemination speed decreases near a $60 \%$ in average, compared to $N_{s}=5$, in which case it would not be the recommended value if applications have a strict requirement about the speed of dissemination.

Figure 4 corresponds to the evaluation of the TrAD protocol. We tested the protocol for the two vehicular densities and the different network loads. Note that no parameter values of the protocol are adjusted in the evaluation since the protocol employs an adaptive beaconing technique integrated into the protocol. The results show the high capacity of the protocol to adapt to different scenarios of operation, which delivers good results even when the scenario becomes more challenging (high density/high network load). However, this protocol involves additional complexity since it requires more computational capacity in each node and additional overhead appended to the beacon messages.

\section{Conclusions and future work}

In this paper, we have provided a baseline study for the performance of two representative dissemination protocols: the Slotted 1-persistence and the TrAD. The study aimed at showing how the performance is highly sensitive to the parameter values employed given a context of operation for the vehicular network. The baseline study serves as an input for a system where the contextual information is identified, with the purpose of changing the parameters of a dissemination mechanism in vehicular scenarios, to improve its performance significantly. In this regard, context information is not limited only to the characteristics of the vehicular traffic, but it can also include the state of the communications network. All in 


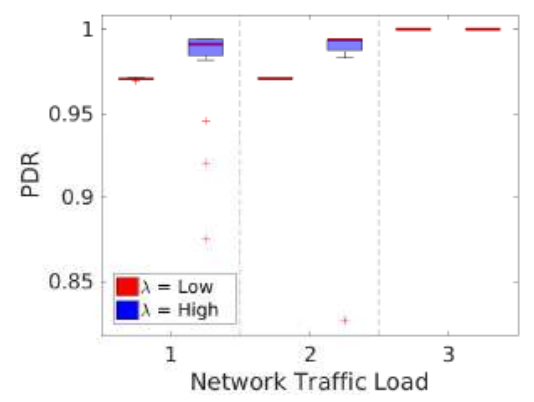

(a) Packet delivery ratio

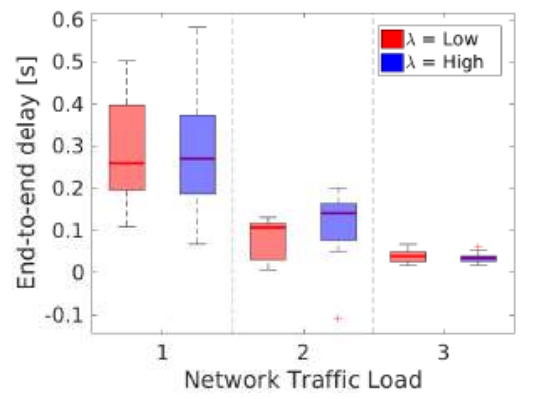

(b) End to end delay

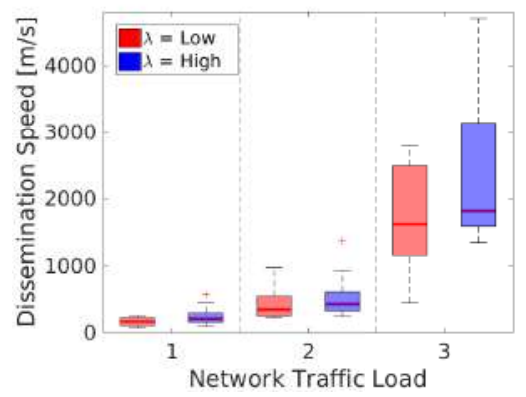

(c) Dissemination speed

Figure 4 TrAD evaluation: different network loads and low/high vehicular density

Table 2 Simulation parameters

\begin{tabular}{ll}
\hline Physical layer & \\
\hline Frequency & $5.89 \mathrm{GHz}$ \\
SimplePathLoss Model & $\alpha=2$ \\
Transmission power & $20 \mathrm{~mW}$ \\
Receiver sensitivity & $-89 \mathrm{dBm}$ \\
Thermal noise & $-110 \mathrm{dBm}$ \\
Antenna type & Monopole \\
\hline Link layer & \\
\hline Bit rate & $6 \mathrm{Mbps}$ \\
Congestion Window & {$[15,1023]$} \\
Slot time & $13 \mu \mathrm{s}$ \\
SIFS & $32 \mu \mathrm{s}$ \\
DIFS & $58 \mu \mathrm{s}$ \\
\hline Messages & \\
\hline Beaconing frequency & $2 \mathrm{~Hz}$ and $10 \mathrm{~Hz}$ \\
Beacon size & $256 \mathrm{bits}$ \\
WSA frequency & $1 \mathrm{~Hz}$ \\
WSA size & $250 \mathrm{bits}$ \\
WSM size & $1024 \mathrm{bits}$ \\
\hline Vehicular traffic & \\
\hline Vehicular density & $15.6,73.4 \mathrm{veh} / \mathrm{km} / \mathrm{ln}$ \\
Max speed & $19 \mathrm{~m} / \mathrm{s}$ \\
Vehicle type & Buses and cars \\
\hline Slotted-1-Persistence & \\
\hline$\tau$ & $5 \mathrm{~ms}$ \\
Range & $500 \mathrm{~m}$ \\
$N_{s}$ & 2,3 and 5,7 \\
\hline TrAD & \\
\hline$\alpha$ & 25 \\
\# Max.Neighbors & $5 \mathrm{~ms}$ \\
Max.Range & \\
\hline & \\
\hline & \\
\hline
\end{tabular}

all, context information is advantageous to make essential decisions about the communications in the VANET and other ad-hoc networks; for example, it serves in the adjustment of the beaconing frequency to guarantee the operation of safety applications.

In our on-going work, we are implementing the scenario classifier based on a machine learning algorithm, so that the system can classify the scenario in which a VANET node operates automatically and in real time.

\section{Acknowledgment}

This work has been partially funded by CONICYT Chile through the RETRACT project ELAC2015/T100761, the National Council of Research and Technology of Mexico [CONACYT] through grant FONCICYT/272278, and the Complex Engineering Systems Institute, ISCI (CONICYT: FB0816).

\section{References}

[1] X. Ma, J. Zhang, X. Yin, and K. S. Trivedi, "Design and analysis of a robust broadcast scheme for VANET safety-related services," IEEE Transactions on Vehicular Technology, vol. 61, no. 1, pp. 46-61, 2012.

[2] B. Hassanabadi and S. Valaee, "Reliable periodic safety message broadcasting in VANETs using network coding," IEEE Transactions on Wireless Communications, vol. 13, no. 3, pp. 1284-1297, 2014.

[3] J. J. Alcaraz, J. Vales-Alonso, and J. Garcia-Haro, "Control-based scheduling with QoS support for vehicle to infrastructure communications," IEEE Wireless Communications, vol. 16, no. 6, pp. 32-39, 2009.

[4] K. D. Kusano and H. C. Gabler, "Comparison of expected crash and injury reduction from production forward collision and lane departure warning systems," Traffic injury prevention, vol. 16, no. sup2, pp. S109-S114, 2015.

[5] G. Tan, Y. Yin, J. Bu, and N. Ding, “An ivc broadcast scheme based on traffic phase for emergency message dissemination at road intersection," in Complex, Intelligent, and Software Intensive Systems (CISIS), 2013 Seventh International Conference on. IEEE, 2013, pp. $59-65$.

[6] Y. Bi, H. Zhou, W. Zhuang, and H. Zhao, Safety Message Broadcast in Vehicular Networks. Springer, 2016.

[7] O. K. Tonguz, N. Wisitpongphan, and F. Bai, "Dv-cast: A distributed vehicular broadcast protocol for vehicular ad hoc networks," IEEE Wireless Communications, vol. 17, no. 2, 2010. 
[8] Y.-C. Tseng, S.-Y. Ni, Y.-S. Chen, and J.-P. Sheu, "The broadcast storm problem in a mobile ad hoc network," Wireless networks, vol. 8, no. 2-3, pp. 153-167, 2002

[9] A. Yáñez, S. Céspedes, and J. Rubio-Loyola, “Cassam: Context-aware system for safety messages dissemination in vanets," in 2018 IEEE Colombian Conference on Communications and Computing (COLCOM). IEEE, 2018, pp. 1-6.

[10] H. Moustafa and Y. Zhang, Vehicular Networks: Techniques, Standards, and Applications, 1st ed. Boston, MA, USA: Auerbach Publications, 2009

[11] R. Kumar, M. Dave et al., "A review of various vanet data dissemination protocols," International Journal of u-and e-Service, Science and Technology, vol. 5, no. 3, pp. 27-44, 2012.

[12] N. Kaur and A. Singh, "A survey on data dissemination protocols used in vanets," International Journal of Computer Applications, vol. 120, no. 23,2015 .

[13] N. Wisitpongphan, O. Tonguz, J. Parikh, P. Mudalige, F. Bai, and V. Sadekar, "Broadcast storm mitigation techniques in vehicular ad hoc networks," IEEE Wireless Communications, vol. 14, no. 6, pp. 84-94, 2007.

[14] M. Li, K. Zeng, and W. Lou, "Opportunistic broadcast of event-driven warning messages in vehicular ad hoc networks with lossy links," Computer Networks, vol. 55, no. 10, pp. 2443-2464, 2011.

[15] A. Galaviz-Mosqueda, S. Villarreal-Reyes, H. Galeana-Zapien, J. Rubio-Loyola, and R. Rivera-Rodriguez, "Genetic tuning of fuzzy rule-based systems for multi-hop broadcast protocols for VANETs," Telecommunication Systems, vol. 63, no. 3, pp. 399-420, 2016. [Online]. Available: "http://dx.doi.org/10.1007/s11235-015-0129-z

[16] Y. Zhu, B. Xu, X. Shi, and Y. Wang, "A survey of social-based routing in delay tolerant networks: Positive and negative social effects," IEEE Communications Surveys \& Tutorials, vol. 15, no. 1, pp. 387-401, 2013.

[17] Y. Cao and Z. Sun, "Routing in delay/disruption tolerant networks: A taxonomy, survey and challenges," IEEE Communications surveys \& tutorials, vol. 15, no. 2, pp. 654-677, 2013.

[18] C. Sommer, D. Eckhoff, and F. Dressler, "Ivc in cities: Signal attenuation by buildings and how parked cars can improve the situation," IEEE Transactions on Mobile Computing, vol. 13, no. 8, pp. 1733-1745, 2014

[19] C. Sommer, S. Joerer, M. Segata, O. K. Tonguz, R. L. Cigno, and F. Dressler, "How shadowing hurts vehicular communications and how dynamic beaconing can help," IEEE Transactions on Mobile Computing, vol. 14, no. 7, pp. 1411-1421, 2015.

[20] R. S. Schwartz, R. R. Barbosa, N. Meratnia, G. Heijenk, and H. Scholten, "A directional data dissemination protocol for vehicular environments," Computer Communications, vol. 34, no. 17, pp. 2057-2071, 2011

[21] B. Tian, K. M. Hou, and H. Zhou, "The traffic adaptive data dissemination (TrAD) protocol for both urban and highway scenarios," Sensors (Switzerland), vol. 16, no. 6, p. 920, jun 2016. [Online]. Available: http://www.ncbi.nlm.nih.gov/pubmed/27338393

[22] M. Hager, L. Wernecke, C. Schneider, and J. Seitz, "Vehicular ad hoc networks: Multi-hop information dissemination in an urban scenwario," in Telecommunications and Signal Processing (TSP), 2015 38th International Conference on. IEEE, 2015, pp. 65-70.

[23] I.-H. Bae, "An intelligent broadcasting algorithm for early warning message dissemination in vanets," Mathematical Problems in Engineering, vol. 2015, 2015.

[24] L. A. Villas, A. Boukerche, G. Maia, R. W. Pazzi, and A. A. Loureiro, "Drive: An efficient and robust data dissemination protocol for highway and urban vehicular ad hoc networks," Computer Networks, vol. 75, pp. 381-394, 2014. [Online]. Available: https://www. sciencedirect.com/science/article/abs/pii/S1389128614003673

[25] S. Bolufé, S. Montejo-Sánchez, C. Azurdia-Meza, S. Céspedes, R. Demos Souza, and E. M. García, "Dynamic Control of Beacon Transmission Rate and Power with Position Error Constraint in Cooperative Vehicular Networks," in 33rd ACM/SIGAPP Symposium On Applied Computing (SAC), Pau, France, Apr 2018.

[26] R. Oliveira, C. Montez, A. Boukerche, and M. S. Wangham, "Reliable data dissemination protocol for VANET traffic safety applications," Ad Hoc Networks, vol. 63, pp. 30-44, 2017.
[27] M. Segata, F. Dressler, and R. L. Cigno, “Jerk beaconing: A dynamic approach to platooning," in Vehicular Networking Conference (VNC), 2015 IEEE. IEEE, 2015, pp. 135-142.

[28] S. A. A. Shah, E. Ahmed, F. Xia, A. Karim, M. Shiraz, and R. M. Noor, "Adaptive beaconing approaches for vehicular ad hoc networks: a survey," IEEE Systems Journal, 2016.

[29] M. Bakhouya, "Broadcasting approaches for mobile ad hoc networks," in High Performance Computing and Simulation (HPCS), 2013 International Conference on. IEEE, 2013, pp. 705-707.

[30] A. Dua, N. Kumar, and S. Bawa, "An energy efficient data dissemination and information retrieval scheme for vanet," in Data Science and Data Intensive Systems (DSDIS), 2015 IEEE International Conference on. IEEE, 2015, pp. 427-434.

[31] S. Poslad, "Context-aware systems," Ubiquitous Computing: Smart Devices, Environments and Interactions, pp. 213-244, 2009.

[32] H. Vahdat-Nejad, A. Ramazani, T. Mohammadi, and W. Mansoor, "A survey on context-aware vehicular network applications," Vehicular Communications, vol. 3, pp. 43-57, 2016. [Online]. Available: http: //www.sciencedirect.com/science/article/pii/S2214209616000036

[33] R. Want, A. Hopper, V. Falcao, and J. Gibbons, "The active badge location system," ACM Transactions on Information Systems (TOIS), vol. 10, no. 1, pp. 91-102, 1992.

[34] K. Abrougui, A. Boukerche, and R. W. N. Pazzi, "Design and evaluation of context-aware and location-based service discovery protocols for vehicular networks," IEEE Transactions on Intelligent Transportation Systems, vol. 12, no. 3, pp. 717-735, 2011.

[35] W. Woernd and R. Eigner, "Collaborative, context-aware applications for inter-networked cars," in Proceedings of the Workshop on Enabling Technologies: Infrastructure for Collaborative Enterprises, WETICE. IEEE, 2007, pp. 180-185. [Online]. Available: http: //ieeexplore.ieee.org/document/4407149/

[36] H. Zarza, H. Karimi, S. Mohammad, S. Y. Hosseini, H. R. Ataeian, and H. Rostami, "Cagfp: context aware geocast forwarding protocol for inter-vehicle communication," in 21st Iranian Conference on Electrical Engineering (ICEE), IEEE, Mashhad, Iran, 2013. [Online]. Available: http://confnews.um.ac.ir/images/41/ conferences/icee2013/1854_6.pdf

[37] N. Alsharif, S. Cespedes, and X. S. Shen, "ICAR: Intersection-based connectivity aware routing in vehicular ad hoc networks," in IEEE International Conference on Communications. IEEE, jun 2013, pp. 1736-1741. [Online]. Available: http://ieeexplore.ieee. org/document/6654769/

[38] R. B. Soares, E. F. Nakamura, C. M. Figueiredo, and A. A. Loureiro, "VCARP: Vehicular Ad-hoc Networks context-aware routing protocol," in Proceedings - IEEE Symposium on Computers and Communications. IEEE, jul 2012, pp. 000 442-000 447. [Online]. Available: http://ieeexplore.ieee.org/document/6249336/

[39] P. Shankar, T. Nadeem, J. Rosca, and L. Iftode, "CARS: Context-aware rate selection for vehicular networks," in Proceedings - International Conference on Network Protocols, ICNP. IEEE, oct 2008, pp. 1-12. [Online]. Available: http: //ieeexplore.ieee.org/document/4697019/

[40] F. Dressler, F. Klingler, C. Sommer, and R. Cohen, "Not All VANET Broadcasts Are the Same: Context-Aware Class Based Broadcast," IEEE/ACM Transactions on Networking, vol. PP, no. 99, pp. 1-14, 2017.

[41] D. Böhmländer, T. Dirndorfer, A. H. Al-Bayatti, and T. Brandmeier, "Context-aware system for pre-triggering irreversible vehicle safety actuators," Accident Analysis and Prevention, vol. 103, pp. 72-84, 2017. [Online]. Available: http://dx.doi.org/10.1016/j.aap.2017.02. 015

[42] Z. Amjad, W. C. Song, K. J. Ahn, and M. Shoaib, "Context aware data aggregation in vehicular ad-hoc networks," in NOMS 2016 - 2016 IEEE/IFIP Network Operations and Management Symposium. IEEE, apr 2016, pp. 1257-1260. [Online]. Available: http://ieeexplore.ieee. org/document/7502998/

[43] S. Yi, W. Lai, D. Tang, and H. Wang, "A context-aware MAC protocol in VANET based on Bayesian Networks," in 9th International Conference on Communications and Networking in China. IEEE, aug 2014, pp. 191-196. [Online]. Available: http://ieeexplore.ieee.org/document/ 7054284/ 
[44] R. Bauza, J. Gozalvez, and J. Sanchez-Soriano, "Road traffic congestion detection through cooperative Vehicle-to-Vehicle communications," in Proceedings - Conference on Local Computer Networks, LCN. IEEE, oct 2010, pp. 606-612. [Online]. Available: http://ieeexplore.iee.org/document/5735780/

[45] L. Skycomp, "Inc. in association with whytney bailey,"," Major highway performance ratings and bottleneck inventory," State of Maryland, Spring, vol. 2009, 2008.

[46] G. Karagiannis, O. Altintas, E. Ekici, G. Heijenk, B. Jarupan, K. Lin, and T. Weil, "Vehicular networking: A survey and tutorial on requirements, architectures, challenges, standards and solutions," IEEE communications surveys \& tutorials, vol. 13, no. 4, pp. 584-616, 2011.

[47] C. Sommer, R. German, and F. Dressler, "Bidirectionally Coupled Network and Road Traffic Simulation for Improved IVC Analysis," IEEE Transactions on Mobile Computing, vol. 10, no. 1, pp. 3-15, January 2011. 